\title{
Coprodutos da agroindústria na alimentação de leitões
}

\author{
Agro-industrial co-products in feed for piglets
}

\begin{abstract}
Rodrigo Mariano Volpato ${ }^{\mathrm{I}}$ Vladimir de Oliveira ${ }^{\mathrm{II}}$ Clóvis Eliseu Gewehr ${ }^{\mathrm{III}}$ Diego Perez Neto
\end{abstract}
\section{RESUMO}

Foi realizado um experimento para determinar a composição química e digestibilidade dos nutrientes e energia do resíduo de batata palha (RBP), resíduo de pipoca doce (RPD), resíduo de biscoito de trigo doce (RBT), e resíduo de biscoito de trigo salgado (RBS) para leitões. Foram determinados os valores de matéria seca $(M S)$, proteína bruta $(P B)$, extrato etéreo $(E E)$, matéria mineral $(\mathrm{MM})$, cálcio $(\mathrm{Ca})$, fósforo $(\mathrm{P})$, sódio $(\mathrm{Na})$ e energia bruta (EB). A digestibilidade dos alimentos foi avaliada utilizando 18 suínos alojados em gaiolas de metabolismo durante dois períodos consecutivos de 12 dias, sendo sete para adaptação e cinco destinados à coleta total de fezes. Os resultados de PB obtidos foram relativamente baixos, sendo 7,2; 6,0; 6,8 e 3,4\% para o $R B P, R P D, R B T$ e RBS, respectivamente. Os conteúdos de EE foram de 39,64; 7,12; 12,40; e 22,28\% para o RBP, RPD, RBT e RBS, respectivamente. Os valores de Ca e $P$ foram baixos e, em todos os coprodutos, inferiores a 0,18\%. Teores elevados de $\mathrm{Na}$ foram encontrados no RBP e RBS $(0,9$ e 1,36\%, respectivamente). A EB foi de 6.065; 4.199; 4.494; e $5.032 \mathrm{kcal} \mathrm{kg}^{-1}$ para RBP, RPD, $R B T$ e RBS, respectivamente. Os valores de ED foram de 5.686; 3.995; 3.916 e $4.953 \mathrm{kcal} \mathrm{kg}^{-1}$, para RBP, RPD, RBT, e RBS e mostraram correlação linear e positiva $\left(r^{2}=0,97 ; P<0,05\right) \mathrm{com}$ o teor de EE dos coprodutos. Estudos adicionais são necessários para estabelecer os teores ótimos de inclusão dos coprodutos analisados nas dietas de suínos. Contudo, os resultados de composição química e digestibilidade dos nutrientes e energia permitem concluir que os resíduos de batata palha, pipoca doce, biscoito de trigo doce e biscoito de trigo salgado possuem potencial para serem utilizados na alimentação de leitões.

Palavras-chave: alimentos alternativos, coprodutos, energia digestivel, nutrição animal, suínos.

\section{ABSTRACT}

One experiment was realized to determine the chemical composition and digestibility of nutrients and energy of

\begin{abstract}
shoestring potatoes residues (SP), sweet popcorn residues (SPC), wheat sweet biscuit residues (WSB) and wheat salted sticks residues (WSS) for piglets. The dry matter (DM), crude protein $(C P)$, ether extract $(E E)$, mineral matter $(M M)$, calcium $(C a)$, phosphorus $(P)$ and sodium (Na) and gross energy (GE) were measured. Eighteen castrated pigs penned in metabolic crates during two consecutive periods of 12 days (seven to adaptation and five to feces collection) were used to evaluate the digestibility's coefficients of feedstuffs. The SP, SPC, WSB, and WSS had relatively low CP values 7.2; 6.0; 6.8 and $3.4 \%$, respectively. The EE contents were 39.64; 7.12; 12.40 and $22.28 \%$ for SP, SPC; WSB and WSS, respectively. All the co-products evaluated had low values of $C a$ and $P$ and anyone had a Ca and P value higher than $0.18 \%$ and the SPC and WSS had high $\mathrm{Na}$ levels $(0.9$ and $1.36 \%$, respectively). The GE were 5.686; 3.995; 3.916; 4.953kcal kg-1 for SP, SPC; WSB and WSS, respectively and showed positive linear correlation $\left(r^{2}=0.97\right.$; $P<0.05)$ with EE levels. Additional studies are necessary to establish the optimal levels of co-products to include in pigs diets. However, the results of chemical composition and digestibility of nutrients and energy indicate that shoestring potatoes residues, sweet popcorn residues, wheat sweet biscuit residues and wheat salted sticks residues have potential to be used in piglet's diets.
\end{abstract}

Key words: animal nutrition, by-product, co-product, digestible energy, pig.

\section{INTRODUÇÃO}

Os preços elevados que o suinocultor seguidamente paga pelo milho e farelo de soja, principais insumos utilizados na alimentação de suínos, contribuem para diminuir a rentabilidade da suinocultura. Inúmeras tecnologias são constantemente testadas visando a propor soluções que possam reverter ou amenizar essa situação.

\footnotetext{
'Departamento de Zootecnia, Universidade Federal de Santa Catarina (UFSC), Florianópolis, SC, Brasil.

ID Departamento de Zootecnia, Universidade Federal de Santa Maria (UFSM), 97105-900, Santa Maria, RS, Brasil. E-mail: vladimir.oliveira@ufsm.br. Autor para correspondência.

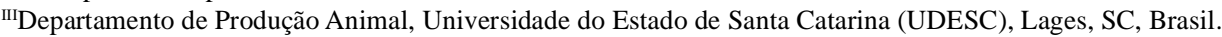
Recebido 22.03.13 Aprovado 31.05.14 Devolvido pelo autor 27.08.14 CR-2013-0391.R1
} 
Nesse contexto, torna-se relevante a avaliação de alimentos alternativos nas dietas de suínos, uma vez que os gastos com a nutrição do rebanho são os que mais impactam os custos de produção (FIALHO, 2009). Alimentos industrializados que se destinam ao consumo humano e que, por algum motivo, são descartados constituem uma categoria que desperta atenção dos pesquisadores. Além das vantagens econômicas, o fornecimento desses coprodutos aos animais também pode ser uma alternativa ao lançamento indiscriminado no meio ambiente (REZZADORI \& BENEDETTI, 2009).

Produtos da indústria alimentícia são eventualmente descartados por estarem quebrados, queimados, amassados e fora do prazo de validade para consumo humano. A batata palha, pipoca doce, biscoito de trigo e o salgadinho são exemplos desses produtos. São constituídos de matérias-primas como farinha de trigo, milho, açúcar, óleo vegetal, entre outras, que são bem aproveitadas pelos suínos, de forma a se supor que tenham algum potencial para uso na alimentação desses animais.

A composição química e os valores energéticos dos alimentos são informações fundamentais no momento da formulação das rações (SAUVANT et al., 2004). Enquanto a composição química permite saber quais os nutrientes que constituem os alimentos, evidenciando alguns de seus limites e potencialidades, os valores energéticos são indicadores da quantidade de energia liberada durante a oxidação metabólica.

Aenergia digestível de um alimento pode ser determinada in vivo pelos métodos direto ou indireto. No método indireto, o alimento teste substitui uma proporção da dieta referência, sendo que o nível de substituição depende das características do alimento teste, podendo inclusive se utilizar mais de um nível de inclusão. Estimar o coeficiente de digestibilidade e valor energético incluindo-se diferentes níveis do alimento teste tem vantagens em relação ao uso de apenas um nível (BAIDOO et al., 1996: KUNRATH et al., 2010). Possibilita gerar estimativas com menor erro padrão e verificar se há interação entre dieta basal e alimento teste (WISEMAN \& COLE, 1987; KUNRATH et al., 2010).

Diante disso, foi realizado um experimento para avaliar a composição química e os coeficientes de digestibilidade dos nutrientes e da energia de resíduo de batata palha (RBP), resíduo de pipoca doce (RPD), resíduo de biscoito de trigo (RBT) e resíduo de biscoito salgado (RBS) para leitões.

\section{MATERIAL E MÉTODOS}

Os quatro coprodutos avaliados foram o resíduo de batata palha (RBP), resíduo de pipoca doce (RPD), resíduo de biscoito de trigo (RBT) e resíduo de biscoito salgado (RBS), todos obtidos de uma indústria alimentícia que tem estes produtos na sua linha de produção. O RBP era constituído por batata frita em óleo e sal. O RPD era a base de milho extrusado e açúcar. O RBT era constituído de rosquinhas elaboradas a base de farinha de trigo. O RBS tinha o formato de palito e apresentava, como principais constituintes, a farinha de trigo e o sal. Todos os resíduos avaliados foram elaborados por indústria de alimentos destinados ao consumo humano. Eram recolhidos no varejo, devido à presença de danos na embalagem e, por ocasião do experimento, estavam dentro do prazo de validade. Foram utilizados 18 suínos castrados provenientes de granja comercial. Antes de serem selecionados para o experimento, os leitões estavam sendo alimentados com dieta formulada para atender as exigências nutricionais da fase. Durante o estudo, os leitões foram alojados individualmente em gaiolas metabólicas por dois períodos consecutivos de 12 dias (sete dias de adaptação à dieta experimental e cinco dias de coleta). Os animais apresentaram pesos médios iniciais de $8,1 \pm 0,75$ e $12,5 \pm 1,1 \mathrm{~kg}$, no primeiro e segundo períodos, respectivamente. Os suínos foram distribuídos em nove tratamentos experimentais, que consistiram de uma dieta referência (sem inclusão dos subprodutos avaliados) e oito dietas teste, contendo níveis crescentes dos coprodutos avaliados (RBP, 15 e 30\%; RPD, 7,5 e 15\%; RBT, 15 e 30\%; e, RBS, 7,5 e $15 \%$ ). Ao total, foram utilizadas 36 observações e quatro repetições por tratamento. $\mathrm{O}$ milho e os coprodutos utilizados nas dietas experimentais foram triturados em moinho de martelos, equipado com peneira de $2 \mathrm{~mm}$ e a mistura das rações foi realizado com auxílio de um misturador com capacidade para $150 \mathrm{~kg}$. A dieta referência foi calculada para atender as exigências nutricionais de energia, aminoácidos, vitaminas e minerais, conforme sugestões do NRC (1998) apresentados na tabela 1. A quantidade de ração fornecida foi ajustada para que o consumo fosse semelhante, com base no peso vivo metabólico $\left(\mathrm{PV}^{0,75}\right)$. O alimento foi distribuído em duas refeições diárias, às 8 e 17 horas e a água disponível à vontade.

O início e o fim da coleta de fezes foram determinados incluindo-se $0,5 \%$ de óxido férrico (Fe2O3) como marcador fecal. As fezes e urina foram coletadas diariamente e, ao final do período experimental, foram pesadas, homogeneizadas e 
Tabela 1 - Ingredientes e composição química analisada da dieta referência (DR) e com inclusão de resíduos de batata palha (RBP), biscoito de trigo doce (RBD), pipoca doce (RPD) e biscoito de trigo salgado (RBS).

\begin{tabular}{|c|c|c|c|c|c|c|c|c|c|}
\hline Ingredientes $(\%)$ & DR & $\begin{array}{c}\text { RBP } \\
15\end{array}$ & $\begin{array}{c}\text { RBP } \\
30\end{array}$ & $\begin{array}{c}\text { RBD } \\
15\end{array}$ & $\begin{array}{c}\text { RBD } \\
30\end{array}$ & $\begin{array}{c}\text { RPD } \\
7,5\end{array}$ & $\begin{array}{c}\text { RPD } \\
15\end{array}$ & $\begin{array}{c}\text { RBS } \\
7,5\end{array}$ & $\begin{array}{c}\text { RBS } \\
15\end{array}$ \\
\hline Milho & 50,47 & 42,90 & 35,33 & 42,90 & 35,33 & 46,68 & 42,90 & 46,68 & 42,90 \\
\hline Farelo de Soja & 22,22 & 18,89 & 15,55 & 18,89 & 15,55 & 20,55 & 18,89 & 20,55 & 18,89 \\
\hline Núcleo* & 22,22 & 18,89 & 15,55 & 18,89 & 15,55 & 20,55 & 18,89 & 20,55 & 18,89 \\
\hline Resíduo & 0,00 & 15,00 & 30,00 & 15,00 & 30,00 & 7,50 & 15,00 & 7,50 & 15,00 \\
\hline Óleo Soja & 4,00 & 3,40 & 2,80 & 3,40 & 2,80 & 3,70 & 3,40 & 3,70 & 3,40 \\
\hline Lisina & 0,44 & 0,37 & 0,31 & 0,37 & 0,31 & 0,41 & 0,37 & 0,41 & 0,37 \\
\hline Metionina & 0,18 & 0,15 & 0,13 & 0,15 & 0,13 & 0,17 & 0,15 & 0,17 & 0,15 \\
\hline Treonina & 0,27 & 0,23 & 0,19 & 0,23 & 0,19 & 0,25 & 0,23 & 0,25 & 0,23 \\
\hline Prom. Cresc.** & 0,20 & 0,17 & 0,14 & 0,17 & 0,14 & 0,19 & 0,17 & 0,19 & 0,17 \\
\hline \multicolumn{10}{|l|}{ Nutrientes (\%) } \\
\hline Matéria seca & 90,68 & 91,51 & 92,34 & 90,92 & 91,15 & 90,96 & 91,23 & 90,78 & 90,87 \\
\hline Proteína bruta & 21,99 & 19,73 & 17,47 & 19,63 & 17,27 & 20,77 & 19,55 & 20,58 & 19,16 \\
\hline Matéria Mineral & 6,47 & 6,01 & 5,54 & 5,66 & 4,85 & 6,07 & 5,66 & 6,19 & 5,92 \\
\hline Matéria Orgânica & 84,21 & 85,50 & 86,80 & 85,26 & 86,30 & 84,89 & 85,56 & 84,59 & 84,95 \\
\hline
\end{tabular}

* Contendo $35 \%$ de PB; $1,4 \%$ de Lisina; $3 \%$ de cálcio; $1,5 \%$ de fósforo; $1 \%$ de sódio. ** Sulfato de Colistina.

amostras foram retiradas para análises químicas. A composição química dos ingredientes, rações, fezes e urina foram analisadas seguindo metodologias descritas em SILVA \& QUEIROZ (2005).

Os coeficientes de digestibilidade (CD) dos nutrientes e energia das rações experimentais foram obtidos de acordo com a fórmula CDNAx $\left.=((\mathrm{Nix}-\mathrm{Nfx}) / \mathrm{Nix})^{* 100}\right)$, em que: CDANx $=\mathrm{CD}$ aparente do nutriente $\mathrm{x}$; NI = Quantidade ingerida do nutriente $\mathrm{x}$; e $\mathrm{NF}=$ Quantidade excretada nas fezes do nutriente $\mathrm{x}$. Posteriormente, foi calculado o $\mathrm{CD}$ dos coprodutos agroindustriais, utilizando uma regressão linear simples, com o nível de inclusão do resíduo e o $\mathrm{CD}$ dos nutrientes das rações contendo o coproduto de interesse, como variáveis independentes e dependente, respectivamente (ADEOLA, 2001).

Os dados obtidos foram submetidos à análise de variância, utilizando no modelo o efeito do animal, período e tratamento. Posteriormente, foi realizado um procedimento de regressão linear utilizando o programa Minitab (MCKENZIE \& GOLDMAN, 1999).

\section{RESULTADOS E DISCUSSÃO}

A composição química dos alimentos avaliados encontra-se na tabela 2. Observase que os teores de proteína bruta (PB) foram relativamente baixos $(<7 \%)$ e inferiores ao milho (9,0\%; ROSTAGNO et al. 2011), por exemplo, um típico alimento energético. As matérias-primas que constituem o RBP, RBT, RPD e o RBS (farinha de trigo, amido de milho, batata, açúcar e óleo, como exemplo) são pobres em proteína, justificando os resultados encontrados. No caso do RPD, nosso resultado é próximo ao encontrado por IMAIZUMI et al. (2006), que foi de 5,35\% de PB.

O conteúdo de extrato etéreo (EE) foi bastante variável entre os coprodutos testados, com o RPD, contendo cerca de 18 e $31 \%$ menos EE que o RBP e RBS, respectivamente. A concentração elevada de EE no RBP e RBS pode ser explicada pelo fato de ser utilizado óleo no processamento (fritura) desses resíduos. Cabe destacar que o teor de EE certamente representará uma restrição para a inclusão do RBP e RBS, pois se recomendam limites máximos de EE em dietas para leitões (MAXWELL \& CARTER, 2001).

Todos os coprodutos apresentaram valores relativamente baixos de cálcio (Ca) $(<0,17 \%)$, característica típica de ingredientes energéticos de origem vegetal (SAUVANT et al., 2004). Contudo, algumas diferenças entre os resíduos foram percebidas, como, por exemplo, a concentração de Ca no RBS foi três vezes superior ao RBP que, por sua vez, apresentou metade do Ca que a média dos demais resíduos.

$\mathrm{O}$ teor de $\mathrm{P}$ foi baixo em todos os coprodutos analisados (média de 0,12\%), o que parece ser característica desses tipos de subprodutos, uma vez que resíduos como o de biscoito/bolacha 
Tabela 2 - Composição química e energia bruta dos resíduos de batata palha (RBP), biscoito de trigo doce (RBT), pipoca doce (RPD) e biscoito de trigo salgado (RBS).

\begin{tabular}{lllll}
\hline Variáveis (\%) & RBP & RBT & RPD & RBS \\
\hline MS & 96,19 & 92,24 & 94,30 & 91,92 \\
$\mathrm{~PB}^{*}$ & 6,91 & 6,26 & 5,66 & 3,10 \\
$\mathrm{EE}^{*}$ & 39,64 & 12,40 & 7,12 & 22,80 \\
$\mathrm{MM}^{*}$ & 3,39 & 1,06 & 1,11 & 2,78 \\
$\mathrm{Ca}^{*}$ & 0,06 & 0,12 & 0,11 & 0,17 \\
$\mathrm{P} *$ & 0,17 & 0,08 & 0,11 & 0,12 \\
$\mathrm{Na}^{*}$ & 0,94 & 0,35 & nd & 1,36 \\
$\mathrm{~EB} *, \mathrm{Kcal} / \mathrm{Kg}$ & 6065 & 4494 & 4199 & 5232 \\
\hline
\end{tabular}

*Valores expressos na matéria seca. MS= matéria seca; $\mathrm{MO}=$ matéria orgânica; $\mathrm{PB}=$ proteína bruta $\mathrm{EB}=$ energia bruta. $\mathrm{nd}=$ não determinado.

e macarrão também contêm pouco $\mathrm{P}$ em sua constituição, apresentando níveis de 0,17 e $0,16 \%$, respectivamente (ROSTAGNO et al., 2011).

Os teores de sódio ( $\mathrm{Na}$ ) foram variáveis entre os coprodutos, embora tanto o RBP como o RBS tivessem conteúdos elevados do mineral (média de $1,1 \%$ ), o que certamente deve ser ponderado quando da inclusão desses subprodutos na dieta de suínos. Considerando que um leitão com $12 \mathrm{~kg}$ de peso vivo precisa ingerir em torno de 1,5 gramas de $\mathrm{Na}$ ao dia (NRC, 1998), estima-se que, com a inclusão de 15 e $10 \%$ de RBP e RBS, respectivamente, praticamente toda a exigência de $\mathrm{Na}$ dessa categoria estaria sendo atendida, ou seja, sem a necessidade de adicionar sal comum. Dessa forma, o teor de sódio poderia definir o limite máximo de inclusão do RBP e RBS nas dietas de leitões.

As diferenças detectadas nos valores de energia bruta (EB) foram positivamente correlacionadas $(r=0,99 ; \mathrm{P}<0,05)$ com o teor de EE e, assim, alimentos com maior teor de EE também tiveram maior conteúdo de EB. Essa mesma tendência é verificada em outros resíduos agroindustriais (ROSTAGNO et al., 2011) e pode ser explicada pelo maior calor de combustão das gorduras em relação à proteínas e carboidratos (NRC, 2012).

Os coeficientes de digestibilidade aparente (CDA) determinados estão na tabela 3 . Todos os resíduos apresentaram CDA da matéria seca (MS) superior a 93\%, indicando que a MS foi bem digerida pelos suínos. Também foi detectada elevada digestibilidade da matéria orgânica (MO), sendo os CDAMO superiores a $96,5 \%$, confirmando que os nutrientes dos coprodutos estudados tiveram boa digestão. Esses resultados são interessantes e evidenciam o potencial RBP, RPD, RBD e RBS na alimentação de suínos, especialmente porque os resultados foram obtidos com leitões na fase inicial de creche, que não apresentam capacidade de digestão totalmente desenvolvida (TOKACH et al., 2003).

Apesar de o conteúdo de PB ser relativamente baixo e os coprodutos avaliados se caracterizarem como alimentos energéticos, a fração digestível da PB foi acima de $90 \%$, exceto para o RPD, cujo CDAPB foi um pouco abaixo $(87,9 \%)$. Valores elevados de CDAPB também são citados por ROSTAGNO et al. (2011) para os resíduos de pão (90\%), biscoito e bolacha $(85 \%)$ e macarrão (88,6\%). Mesmo o RBP, cuja matéria-prima básica é a batata, que contém proteína de baixa digestibilidade (TUŚNIO et al., 2011), apresentou CDAPB elevado (90\%), possivelmente devido ao efeito positivo do calor (fritura) na digestibilidade da proteína.

$\mathrm{Na}$ tabela 4, estão apresentadas as equações usadas para determinar os valores energéticos, bem como a ED dos coprodutos estudados. O intercepto $\left(3.320 \mathrm{kcal} \mathrm{kg}^{-1}\right)$ representa a ED da dieta basal, enquanto o coeficiente angular da reta indica o incremento na ED da dieta a cada $1 \%$ de inclusão do alimento teste (x). Resolvendo a equação para $100 \%$ de inclusão do alimento teste, o resultado será a ED (y) do alimento teste, ou seja, coproduto. As equações foram lineares $(\mathrm{P}<0,05)$, indicando que os valores de ED dos subprodutos não foram influenciados pelos níveis de inclusão. A diferença entre a ED dos alimentos pode ser explicada, em

Tabela 3 - Coeficientes de digestibilidade aparente (CDA) de resíduos de batata palha (RBP), biscoito de trigo doce (RBT), pipoca doce (RPD) e biscoito de trigo salgado (RBS).

\begin{tabular}{lclll}
\hline Variáveis & RBP & RBT & RPD & RBS \\
\hline CDAMS & $93,0( \pm 3,9)$ & $97,0( \pm 0,8)$ & $95,0( \pm 10,6)$ & $95,6( \pm 2,3)$ \\
CDAMO & $97,0( \pm 0,9)$ & $97,8( \pm 0,5)$ & $96,6( \pm 3,5)$ & $97,5( \pm 1,8)$ \\
CDAPB & $90,0( \pm 2,5)$ & $93,4( \pm 3,9)$ & $87,9( \pm 11,9)$ & $90,7( \pm 8,5)$ \\
CDAEB & $91,5( \pm 3,9)$ & $86,9( \pm 0,3)$ & $93,7( \pm 13,4)$ & $94,0( \pm 8,4)$ \\
\hline
\end{tabular}

$\mathrm{MS}=$ matéria seca; $\mathrm{MO}=$ matéria orgânica; $\mathrm{PB}=$ proteína bruta; $\mathrm{EB}=$ =nergia bruta. Valores entre parênteses são erro padrão da média.

Ciência Rural, v.45, n.1, jan, 2015. 
Tabela 4 - Equação de regressão linear e valor de energia digestível de resíduos de batata palha (RBP), biscoito de trigo doce (RBT), pipoca doce (RPD) e biscoito de trigo salgado (RBS).

\begin{tabular}{lcll}
\hline Alimento & Parâmetros da equação regressão & ED $\left(\mathrm{kcal} \mathrm{kg}^{-1}\right)$ & \multicolumn{1}{c}{$\mathrm{R}^{2} *$} \\
\hline RBP & $\mathrm{Y}=3.320( \pm 41,8)+22,0( \pm 2,1)$ & $5.520( \pm 177,0)$ & 0,91 \\
RBT & $\mathrm{Y}=3.320( \pm 2,7)+5,9( \pm 0,1)$ & $3.910( \pm 11,9)$ \\
RPD & $\mathrm{Y}=3.320( \pm 3,3)+6,3( \pm 0,3)$ & $3.950( \pm 30,6)$ & 0,99 \\
RBS & $\mathrm{Y}=3.320( \pm 3,6)+16,3( \pm 0,1)$ & $4.950( \pm 34,1)$ & 0,97 \\
\hline
\end{tabular}

$* \mathrm{R}^{2}=$ coeficiente de determinação.

grande parte, pelo conteúdo de extrato etéreo, pois se verificou associação positiva $(\mathrm{r}=0,97 ; \mathrm{P}<0,05)$ entre os conteúdos de ED e gordura. Todos os resíduos mostraram valores de energia próximos ou superiores ao do milho (ROSTAGNO et al., 2011), por exemplo, um típico alimento usado na dieta de suínos como fonte energética.

Os resultados obtidos no presente estudo demonstram que o resíduo de batata palha (RBP), resíduo de pipoca doce (RPD), resíduo de biscoito de trigo doce (RBT) e resíduo de biscoito de trigo salgado (RBS) apresentam composição química e nutricional que viabilizam a utilização na dieta de suínos. Sugerese que experimentos sejam realizados para determinar o teor ideal de inclusão dos resíduos avaliados nas diferentes fases de criação dos suínos, levando-se em conta as limitações individuais de cada resíduo.

\section{CONCLUSÃO}

Os resultados de composição química e digestibilidade dos nutrientes e energia permitem concluir que os resíduos de batata palha, pipoca doce, biscoito de trigo doce e biscoito de trigo salgado possuem potencial para serem utilizados na alimentação de leitões.

\section{COMITÊ DE ÉTICA}

$$
\text { PROTOCOLO } 5962011 .
$$

\section{AGRADECIMENTOS}

Ao Conselho Nacional de Desenvolvimento Científico e Tecnológico $(\mathrm{CNPq})$ pela bolsa de iniciação científica concedida ao primeiro autor (PIBIC/CNPq - BIP/UFSC 2011/2012). À Boccone Alimentos Ltda pela doação dos co-produtos avaliados.

\section{REFERÊNCIAS}

ADEOLA, O. Digestion and balance techniques in pigs. In: LEWIS, A.J.; SOUTHERN, L.L. (Ed.). Swine nutrition. 2.ed. Boca Raton: CRC, 2001. p.903-916.
BAIDOO, S.K. et al. The digestible energy value of canola oil for growing pigs as measured by level of inclusion. Animal Feed Science Technology, v.62, n. 2 - 4, p.111-119, 1996. Disponível em <http://dx.doi.org/10.1016/S0377-8401(96)00994-7>. Acesso em: 15 mar. 2013. doi: 10.1016/S0377-8401(96)00994-7.

FIALHO, E.T. Alimentos alternativos para suínos. Lavras: UFLA. 2009. 232p.

IMAIZUMI, H. et al. Fontes protéicas e de amido com diferentes degradabilidades ruminais para alimentar vacas leiteiras. Pesquisa Agropecuária Brasileira, Brasília, v.41, n.9, p.1413-1420, 2006. Disponível em: <http://www.scielo.br/pdf/pab/v41n9/ a10v41n9.pdf>. Acesso em: 15 mar. 2013. doi: 10.1590/S0100204X2006000900010

KUNRATH, M.A. et al. Metodologias de avaliação do valor nutricional do farelo de arroz desengordurado para suínos. Pesquisa Agropecuária Brasileira, v.45, n.10, p.11721179, 2010. Disponível em: <http://www.scielo.br/pdf/pab/ v45n10/17.pdf>. Acesso em: 15 mar. 2013. doi: 10.1590/S0100204X2010001000017

MAXWELL, C.V.; CARTER, S. Feeding the weaned pig. In: LEWIS, A.J.; SOUTHERN, L.L. (Ed.). Swine nutrition. 2.ed. Boca Raton: CRC, 2001. p.903-916.

MCKENZIE, J.; GOLDMAN, R.N. The student edition of Minitab for Windows manual: release 12. Belmont: AddisonWesley Longman, Softcover, 1999. 592p.

NUTRIENT REQUIREMENTS OF SWINE (NRC). 10.ed. Washington, DC: National Academy, 1998. 189p.

NUTRIENT REQUIREMENTS OF SWINE (NRC). 11.ed. Washington, DC: National Academy, 2012. 400p.

REZZADORI, K.; BENEDETTI, S. Proposições para valorização de resíduos do processamento do suco de laranja. In: INTERNATIONAL WORKSHOP ADVANCES IN CLEANER PRODUCTION, 2009, São Paulo. Anais... São Paulo: UNIP, 2009. p.1-11. Acessado em 15 mar. 2013. Online. Disponível em: <http://www.advancesincleanerproduction.net/second/english/ site/downloads.html>.

ROSTAGNO, H.S. et al. Tabelas brasileiras para aves e suínos: composição de alimentos e exigências nutricionais. 3.ed. Viçosa: UFV, 2011. 252p.

SAUVANT, D. et al. Tables of composition and nutritional value of feed materials. Wageningen: Academic Publishers/ INRA editions, 2004. 304p. 
SILVA, D.J.; QUEIROZ, A.C. Análise de alimentos: métodos químicos e biológicos. 3.ed. Viçosa: UFV, 2005. 235p.

TOKACH, M.D. et al. Nutritional requirements of weaned pig. In: PLUSKE, J.R. et al. (Ed.). Weaning the pig. Concepts and concepts and consequences. Wageningen: Academic Publishers, 2003. p. $259-271$.

TUŚNIO, A. et al. Response of young pigs to feeding potato protein and potato fibre - nutritional, physiological and biochemical parameters. Journal of Animal and Feed Sciences, v.20, n.3, p.361-378. 2011. Disponível em: <http://www.ifzz.pl/en/ publications/journal-of-animal-and-feed-sciences/contents-from2006-yr/vol-20-2011-r/no-3?sobi2Task=sobi2Details\&catid=72 \& sobi2Id=377>. Acesso em: 15 mar. 2013.

WISEMAN, J.; COLE, D.J.A. The digestible and metabolisable energy of two fat blends for growing pigs as influenced by level of inclusion. Animal Production, v.45, p.117-122, 1987.

Ciência Rural, v.45, n.1, jan, 2015. 